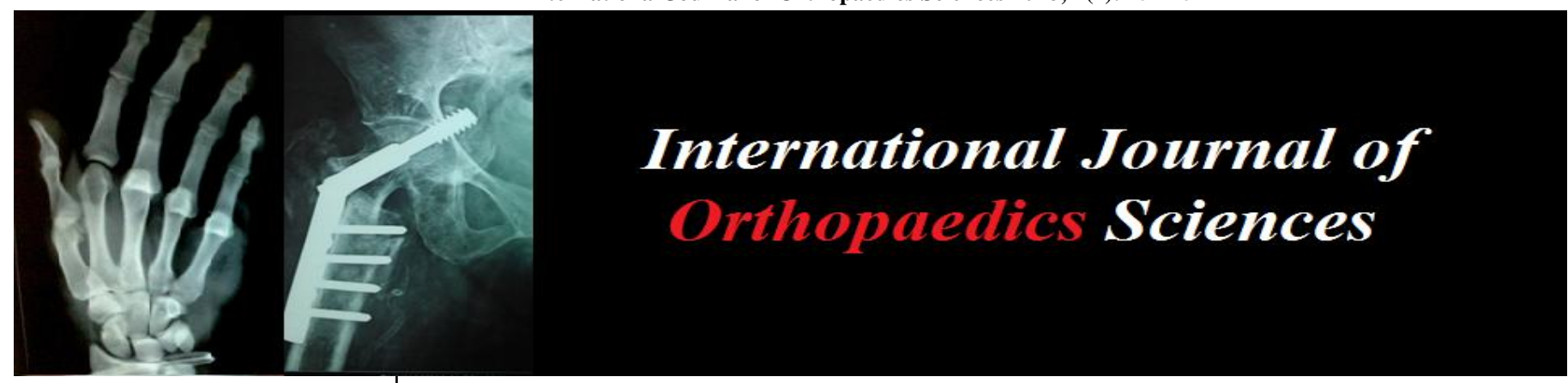

ISSN: $2395-1958$

IJOS 2018; 4(4): 454-457

(C) 2018 IJOS

www.orthopaper.com

Received: 16-08-2018

Accepted: 18-09-2018

Dr. Romeet Mukherjee

PG Resident, Department of Orthopedics Sri Ramachandra Institute of Higher Education and Research, Chennai, Tamil

Nadu, India

Dr. Muthu Manickam

Assistant Professor, Department of Orthopedics Sri Ramachandra Institute of Higher Education and Research, Chennai, Tamil Nadu, India

Dr. N Jambu

Professor, Department of

Orthopedics Sri Ramachandra Institute of Higher Education and Research, Chennai, Tamil Nadu, India
Correspondence

Dr. Muthu Manickam

Assistant Professor, Department of Orthopedics Sri Ramachandra Institute of Higher Education and Research, Chennai, Tamil Nadu, India

\section{Management of acetabular wall fractures with hip dislocation: A case series}

\author{
Dr. Romeet Mukherjee, Dr. Muthu Manickam and Dr. N Jambu
}

DOI: https://doi.org/10.22271/ortho.2018.v4.i4e.44

\section{Abstract}

Introduction: Posterior wall fractures are the most common type of acetabular fractures. Comminuted fractures with extensions to acetabular roof are associated with poor prognosis. Accurate evaluation of resuting union and likelihood of future arthritis or AVN of femoral head are also hindered.

Materials and methods: We report a case series of 9 cases with posterior wall acetabulum fractures associated with posterior dislocation of femoral head treated with internal fixation of posterior wall by three step reonstruction-1)open reduction of dislocation, 2)preservation of soft tissues and reconstruction of marginally impacted and osteochondral fragments using screws, 3)final reinforcement with buttress plating. Functional outcome was evaluated according to D'aubigne and Postel criteria and radiological outcome by criteria of Matta.

Results: According to criteria of Matta $44 \%$ had excellent, $34 \%$ had good and $22 \%$ had fair results.

According to D'aubigne and Postel criteria 50\% had excellent results, 37.5\% had good results, $12.5 \%$ had fair results. None of the cases had heterotrophic ossification. Wound site infection eas the only complication encountered.

Conclusion: We conclude that this three step rconstruction procedure in management of posterior wall acetabular fracture with posterior dislocation of femoral head results in satisfactory functional and radiological outcome without complications.

Keywords: acetabulam, posterior wall, management

\section{Introduction}

Posterior wall fractures are the most common type of acetabular fractures, accounting for approx. $35-47 \%$ of such fractures. Comminuted fractures with more than 2 fragments or extensions to the acetabular roof are co related with poor prognosis. Soft tissues are frequently detached from fracture fragments at the time of injury or during surgery, in addition to that after surgery it is difficult to know the exact quality of reduction and congruity of articular surface due to its complex 3 dimensional shape. Accurate evaluation of resulting union and possibility of future osteoarthritis and differentiating between AVN of femoral head and true post traumatic arthritis are also hindered.

We report 9 cases of internal fixation of posterior wall and proximal femur dislocation by three step reconstruction

1. Open reduction of dislocation.

2. Preservation of attached soft tissues and reconstruction marginally impacted and osteochondral fragments using screws.

3. Final reinforcement with buttress plating.

\section{Methods}

9 patients with posterior wall fractures and dislocation of proximal femur was treated in our institution from 2016-2018. The surgical indications were femoral head subluxation, dislocation, $>50 \%$ involvement of posterior wall, $>2 \mathrm{~mm}$ displacement of fracture fragments in AP view of pelvis without extremity in traction. Our protocol for acetabular fractures included AP view of xrays and 2D and 3D CT scan of pelvis. All fractures were classified after discussing with senior consultants. All 9 cases were male patients. The mean age was 26 years (range 20-50 years). Mode of injury for all was RTA. 
All fractures were associated with hip dislocation. All fractures were classified according to Judet and Letournel system. All patients were treated in casualty with haemodynamic stabilization and was put on upper tibial skeletal traction. No closed reduction was performed on any of the patients. Only one of the patients had an associated sciatic nerve injury.

\section{Surgical Technique}

Three Step reconstruction

1. Open reduction of dislocation - A Kocher Langenbach approach was used. Incision was made. The fascia lata was incised and the gluteus maximus was split and the head was found to be dislocated posteriorly with capsular tear in all cases. The head was relocated inside the acetabular socket using standard reduction manoeuvres.

2. Reduction of articular fragments and fixation with screws-cleaning of soft tissue debris between fracture fragments and preservation of attached available capsular soft tissues. The osteochondral free fragments were removed and extent of marginal impaction was identified by applying gentle traction at the hip joint. These impact posterior wall fragments and their attached capsular ligaments were reduced and held with pointed ball spike. Then temporary $\mathrm{k}$ wires were applied. Then these were fixed with screws. (Cannulated cancellous screws/ headless compression screws). Very small fragments were discarded. Cancellous bone grafts were used to fill up defects if any.

3. Butress Fixation: Then these fragments were then buttressed with plates and appropriate screws. (Synthes pelvic plates). These plates were slightly under contoured so as to provide compression to the posterior wall.

Intra operative fluoroscopic images were used finally to check the accuracy of reduction and position of the screws and the implant.

\section{Post operative protocol}

All patients were started on in bed mobilization from post operative day one. Patients were mobilized on non weight bearing walking with walker support from post operative day 3 and were administered with tab. osteofos $70 \mathrm{mg}$ once daily for 3 months to prevent AVN.

\section{Clinical Grade}

The clinical grade was based on the system of D'aubigne and Postel and as modified by Matta. The grading system includes pain, gait, Range of motion with assigning a maximum of 6 points for each parameter. The 3 parameters values are then added and the total is classified as Excellent (18 points), Very good (17 points), good (15/16 points), Fair (13/14 points) or poor(less than 13 points).

\section{Radiological grade}

Post operative radiographs were used evaluate the congruency of hip and reductional quality. The radiological criteria after open reduction and internal fixation was based on the gap remaining at fracture site after reduction: anatomic $(0-1 \mathrm{~mm})$, good $(2-3 \mathrm{~mm})$ and poor $(>3 \mathrm{~mm})$. The radiological grade at the last follow up based on criteria of Matta: excellent (a normal appearing hip joint), $\operatorname{good}(\operatorname{mild}$ changes with minimal sclerosis joint narrowing less than $1 \mathrm{~mm}$ ), fair (intermediate changes with moderate sclerosis and joint narrowing less than $50 \%$ ) and poor (advanced stages).

\section{Results}

\section{Clinical grades}

The D'Aubigne and Postel scores at final follow up visit were as follows: excellent in 5 patients $(50 \%)$, very good in 3 patients $(37.5 \%)$, fair in 1 patient $(12.5 \%)$.

\section{Radiological grades}

On the basis of gap after the surgery 5 patients had anatomic reduction $(55 \%), 3$ patients had good reduction $(33 \%), 1$ had poor reduction $(12 \%)$. According to radiological criteria of Matta 4 patients had excellent results (44.4\%) 3 had good results $(33 \%)$ and $2(22 \%)$ showed fair results.

\section{Complications}

Out of all the cases operated 2 cases had wound site superficial infection. These patients required wound debridement.1 case had sciatic nerve injury which was identified pre operatively which completely recover by 4 weeks time. There was no heterotrophic ossification or avascular necrosis of femoral head in our study.

\section{Case Illustrations}

Case 1

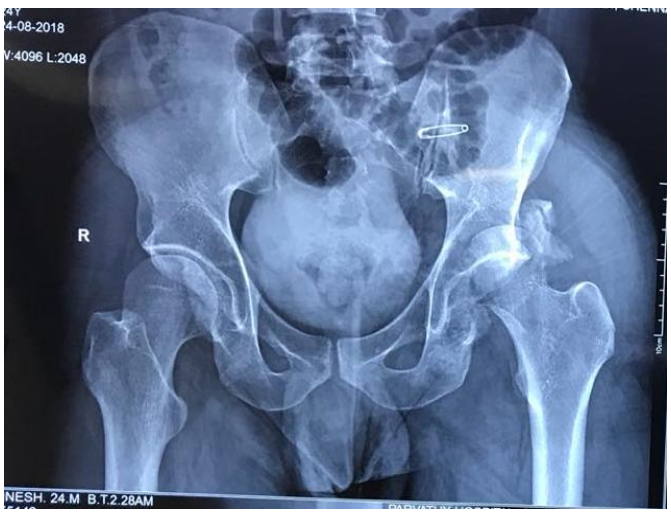

Pre Op Radiograph

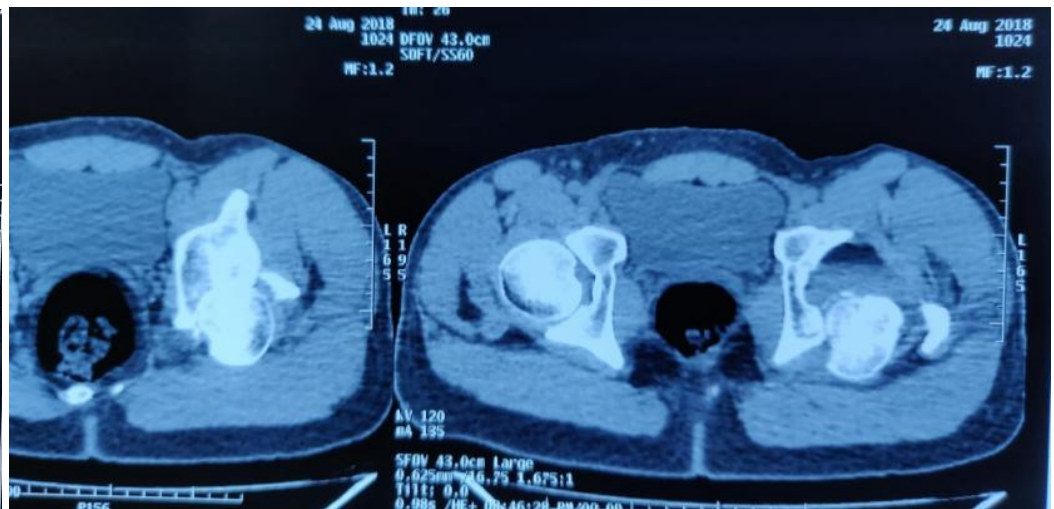

Axial Cut Ct Images 


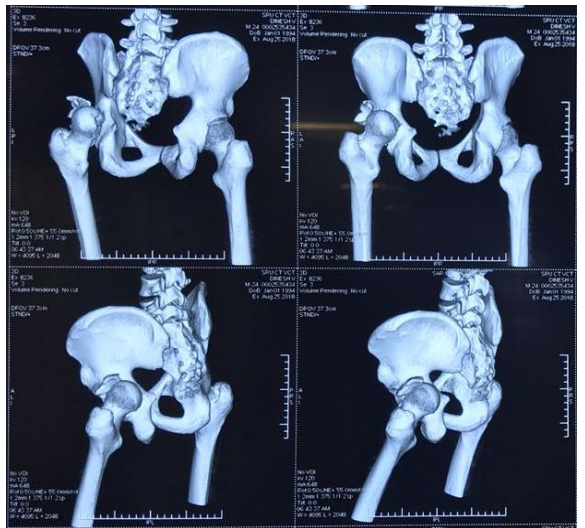

3D ct image

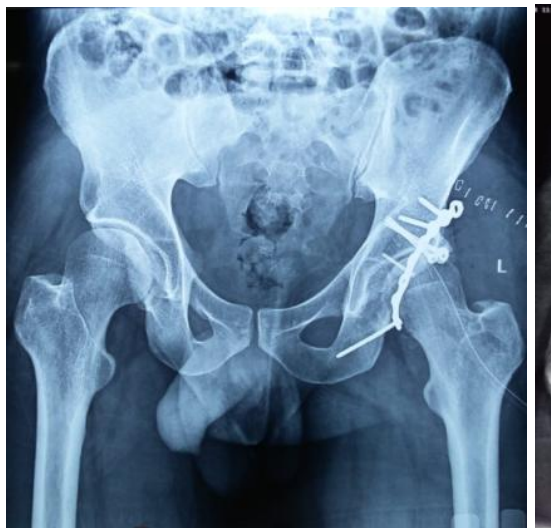

post op xray.

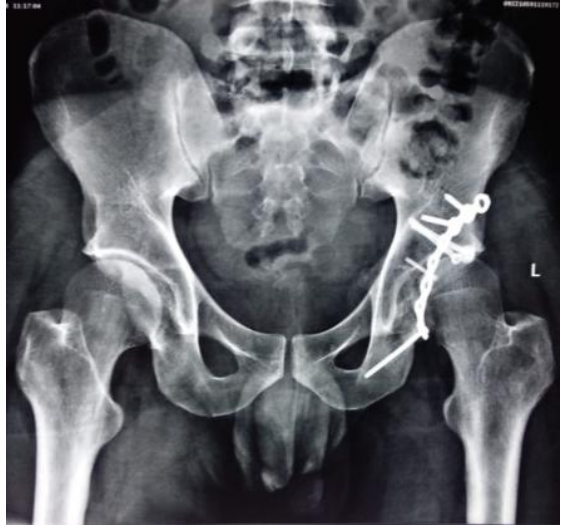

1 year follow up xray

Case 2

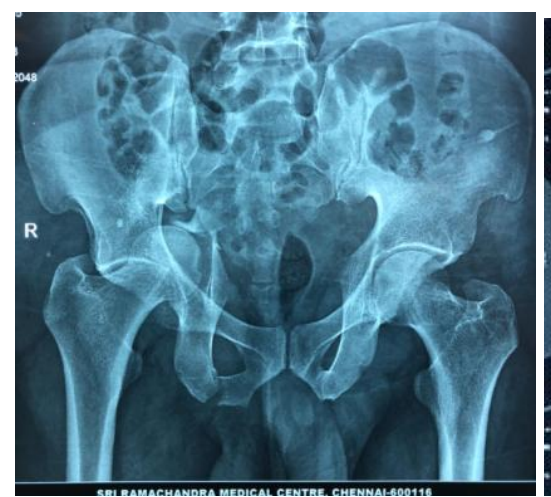

Pre op radiograph

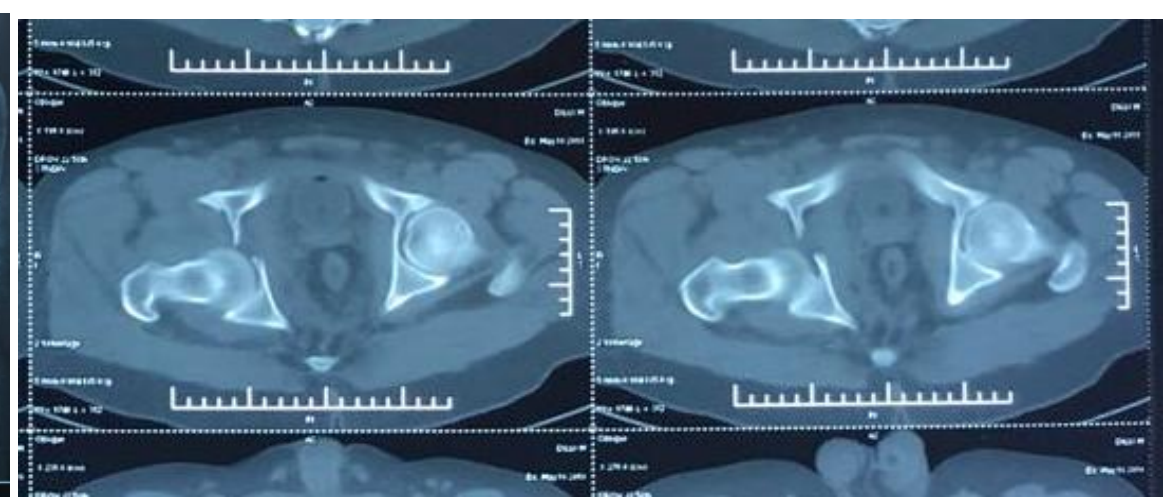

axial ct images

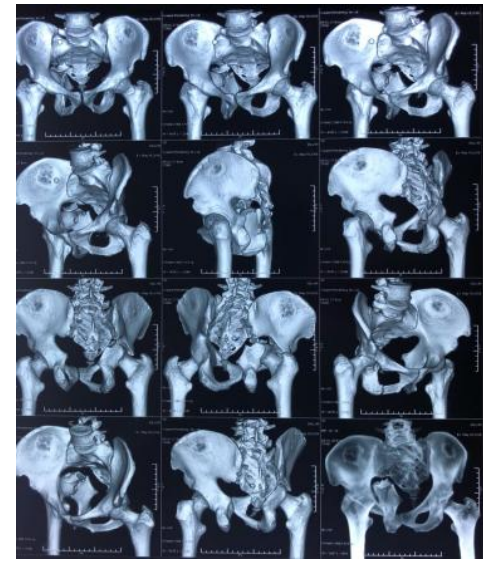

3D ct images

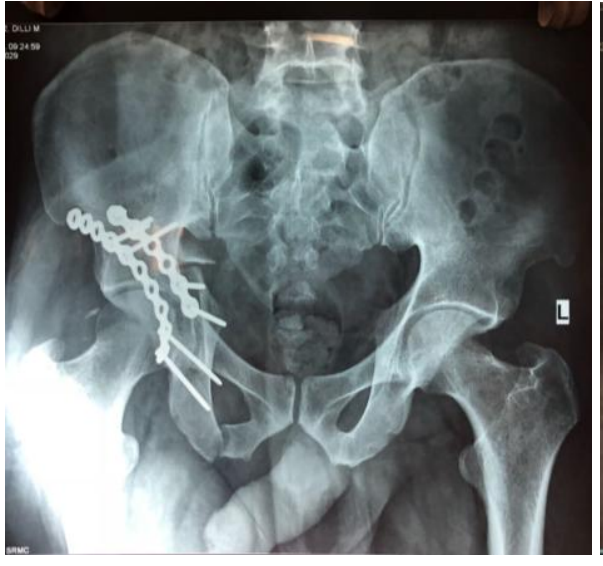

post op xray

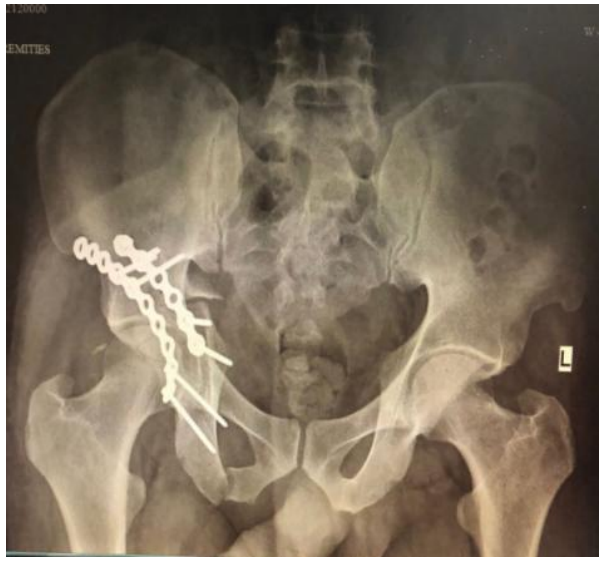

6 months post op

\section{Discussion}

A number of studies ${ }^{[1-3,4,5]}$ have found high rates of poor outcomes and surgical complications for posterior wall fractures of acetabulum when single surgeon performed the surgery. For experienced surgeons the incidence of fair to poor long term results has ranged from 19-25\% ${ }^{[1-3]}$. For inexperienced surgeons who occasionally performed acetabular surgery this was $55-56 \%{ }^{[4,5]}$. Good to excellent results were reported in the range of $74-76 \%{ }^{[2,6-9]}$ in few studies, but expertise of the surgeons were not mentioned. We believe severity of fracture and surgical skill were the main reasons for the lower number of good and excellent outcomes. A lot of techniques have been employed to expose the acetabulum. Baumgartner ${ }^{[17]}$ reported that prone position of Kocher-Langenbach approach was preferred if there was extensive posterior wall fracture. In prone postion there is decreased risk of stretch injury to the sciatic nerve. However prone position is not possible in few patients with facial injuries or open abdominal wounds. In our cases series we used a Kocher Langenbach approach with the patient in lateral decubitis position.

Post operative xrays are important for evaluation of functional and radiological outcome.55\% of our patients had anatomical reduction and $33 \%$ had good reduction. However according to criteria of Matta $44 \%$ had excellent results 2 years post surgery.

In our study we found out that posterior wall fractures which are most commonly associated with posterior dislocation of hip are best treated with 3 step reconstruction with using bone graft for the commented part to reduce mal union and nonunion. We expect that few patients will develop osteoarthritis but this 3 step management procedure will provide a good base for future Total Hip Arthoplasty at a later date. The value of AVN according to few studies has been reported as 3- 
$10 \%$.However true rate is difficult to be obtained. The incidence of sciatic nerve palsy associated with acetabular fracture is also as high as $16 \%$ and it increases to $40 \%$ when there is posterior dislocation of femoral head.

We had one case of preoperative sciatic nerve injury which fully recovered over the following weeks. There were no cases of heterotrophic association. We encountered 2 cases of superficial wound infection which required wound debridement and 4 weeks of antibiotic coverage after culture and sensitivity.

\section{Conclusion}

Hence we conclude that this 3 step reconstruction technique in management of posterior wall fractures of acetabulum associated with posterior dislocation of femoral head results in satisfactory functional and radiological outcome without complications.

\section{References}

1. Letournel E, Judet R. Fractures of the acetabulum. 2nd ed. Berlin: Springer-Verlag, 1993.

2. Matta JM. Fractures of the acetabulum: accuracy of reduction and clinical results in patients managed operatively within three weeks aer the injury. J Bone Joint Surg. Am. 1996; 78(11):1632-45.

3. Mayo KA. Open reduction and internal xation of fractures of the acetabulum: results in 163 fractures. Clin Orthop Relat Res. 1994; (305):31-7.

4. Kaempffe FA, Bone LB, Border JR. Open reduction and internal fixation of acetabular fractures: heterotopic ossification and other complications of treatment. J Orthop Trauma. 1991; 5(4):439-45.

5. Wright $\mathrm{R}$, Barrett $\mathrm{K}$, Christie MJ, Johnson KD. Acetabular fractures: long-term follow-up of open reduction and internal xation. J Orthop Trauma. 1994; 8(5):397-403.

6. Kumar A, Shah NA, Kershaw SA, Clayson AD. Operative management of acetabular fractures: a review of 73 fractures. Injury. 2005; 36(5):605-12.

7. Chiu FY, Chen CM, Lo WH. Surgical treatment of displaced acetabular fractures: 72 cases followed for 10 (6-14) years. Injury. 2000; 31(3):181-5.

8. Deo SD, Tavares SP, Pandey RK, El-Saied G, Willett KM, Worlock PH. Operative management of acetabular fractures in Oxford. Injury. 2001; 32(7):581-6.

9. Petsatodis G, Antonarakos P, Chalidis B, Papadopoulos P, Christoforidis J, Pournaras J. Surgically treated acetabular fractures via a single posterior approach with a follow-up of 2-10 years. Injury. 2007; 38(3):334-43.

10. Murphy D, Kaliszer M, Rice J, McElwain JP. Outcome after acetabular fracture: prognostic factors and their inter- relationships. Injury. 2003; 34(7):512-7.

11. Liebergall M, Moshei R, Low J, Goldvirt M, Matan Y, Segal D. Acetabular fractures: clinical outcome of surgical treat- ment. Clin Orthop Relat Res. 1999; (366):205-16.

12. Moed BR, McMichael JC. Outcomes of posterior wall frac- tures of the acetabulum: surgical technique. J Bone Joint Surg Am. 2008; 90(2):87-107.

13. Moed BR, WillsonCarr SE, Watson JT. Results of operative treatment of fractures of the posterior wall of the acetabulum. J Bone Joint Surg. Am. 2002; 84(5):7528.

14. Johnson EE, Matta JM, Mast JW, Letournel E. Delayed re- construction of acetabular fractures 21-120 days following injury. Clin Orthop Relat Res. 1994; (305):2030.

15. Matta JM, Merritt PO. Displaced acetabular fractures. Clin Orthop Relat Res. 1988; (230):83-97.

16. Helfet DL, Schmeling GJ. Management of complex acetabular fractures through single nonextensile exposures. Clin Orthop Relat Res. 1994; (305):58-68.

17. Baumgaertner MR. Fractures of the posterior wall of the acetabulum. J Am Acad. Orthop Surg. 1999; 7:54-65. 